\title{
Parámetros bioquímicos y morfométricos vinculados a la dentición de búfalas. Relaciones con el número de incisivos
}

\author{
Hernando, J. ${ }^{1}$; Koza, G.A. ${ }^{1}$; Konrad, J.L. ${ }^{2}$; D.Luzio, K.M. ${ }^{1}$; Mussart, N.B. ${ }^{3}$ \\ ${ }^{1}$ Cátedra de Fisiología; ${ }^{2}$ Cátedra de Teriogenología, Facultad de Ciencias Veterinarias, \\ Universidad Nacional del Nordeste (UNNE), Cabral 2139, Corrientes, Argentina. \\ ${ }^{3}$ Cátedra Fisiología Animal, Fac. Cs. Exactas, UNNE. E-mail: kozagaby@gmail.com
}

\begin{abstract}
Resumen
Hernando, J.; Koza, G.A.; Konrad, J.L.; D.Luzio, K.M.; Mussart, N.B.: Parámetros bioquímicos y morfométricos vinculados a la dentición de búfalas. Relaciones con el número de incisivos. Rev. Vet. 30: 2, XX-XX, 2019. La clasificación de las etapas productivas y la salud de un animal o del rodeo pueden establecerse por los cambios observables en los dientes incisivos y la determinación de los valores hematológicos. Este trabajo tuvo como objetivo evaluar el efecto de los cambios del número de incisivos permanentes sobre parámetros morfométricos y bioquímicos sanguíneos relacionados al metabolismo óseo en animales de 32 meses de edad. La investigación fue realizada en Corrientes, Argentina. Búfalas Murrah de 29 meses de edad (n=30), con dentadura decidua completa, fueron pesadas y mantenidas por 90 días en pasturas naturales. $\mathrm{Al}$ final del período se evaluó el número de incisivos permanentes erupcionados, la cantidad y nivel de erupción de piezas dentales, peso (PV), perímetro torácico (PT) y condición corporal (CC), tomándose muestras de sangre para determinar los valores de calcio, fósforo, magnesio y fosfatasa alcalina. La información obtenida fue analizada mediante estadística descriptiva, análisis de la varianza y correlación mediante el test de Pearson. El 37\% mostró dos dientes, el 16\% uno (derecho o izquierdo) y el 47\% no presentó incisivos permanentes. Los valores de PV $(\mathrm{kg})$ y PT $(\mathrm{cm})$ para los individuos con incisivos deciduos, con uno o dos incisivos permanentes, fueron PV: 425,36 $\pm 16,13$; $456 \pm$ 17,67 y 426,64 \pm 12,91 (p = 0,462) y PT: $190 \pm 7,66 ; 197,20 \pm 4,76 ; 189,91 \pm 8,9(\mathrm{p}=$ $0,187)$ respectivamente, sin ser estadísticamente significativos. La magnesemia fue significativamente mayor en los animales sin incisivos y con un diente, comparados con aquellos con dos dientes erupcionados $(r=-0,45 ; \mathrm{p}<0,05)$. Ninguna de las otras variables bioquímicas fueron influenciadas por la cantidad de piezas dentales ( $p \geq 0,05)$. La condición corporal, así como las variables morfométricas y bioquímicas exploradas, no se vieron afectadas por las modificaciones de la cronometría dental, a excepción del magnesio sérico.
\end{abstract}

Palabras clave: búfalo, erupciones dentales, bioquímica sanguínea, morfometría.

Recibido: enero 2019 / Aceptado: junio 2019

\begin{abstract}
Hernando, J.; Koza, G.A.; Konrad, J.L.; D.Luzio, K.M.; Mussart, N.B.: Biochemical and morphometrical parameters associated with dentition in water buffaloes. Relation with the number of incisors. Rev. Vet. 30: 2, XX-XX, 2019. The classification of the productive stages and health of an animal or the herd can be established by changes
\end{abstract}


in the incisor teeth and the determination of hematological values. The aim of this work was to evaluate the effect of changes in the number of permanent incisors on morphometric and blood parameters related to bone metabolism in 32-month-old animals. The assay was performed in Corrientes, Argentina, where 30 Murrah females of 29-monthsold with complete deciduous teeth were weighed and maintained for 90 days on natural pastures. At the end of the assay, the number of erupted permanent incisors and the amount and level of eruption of the dental pieces, were evaluated. Weight (PV), thoracic perimeter (PT) and body condition (CC) were recorded. Blood samples were taken to determine the values of calcium, phosphorus, magnesium and alkaline phosphatase. The information was analyzed with descriptive statistics, analysis of variance and correlation using the Pearson's test. The $37 \%$ of the animals showed two teeth, $16 \%$ one (right or left) and $47 \%$ did not have permanent incisors. The values of PV $(\mathrm{kg})$ and PT $(\mathrm{cm})$ for individuals with deciduous incisors, with one or pair permanent incisors, were $425.36 \pm$ 16.13; $456 \pm 17.67$; and $426.64 \pm 12.91(\mathrm{p}=0.462)$ and $190 \pm 7.66 ; 197.20 \pm 4.76$; and $189.91 \pm 8.9(\mathrm{p}=0.187)$ respectively, without statistical significance. Magnesemia was significantly higher in animals without incisors and with one tooth, compared to those with two erupted teeth $(r=-0.45 \mathrm{p}<0.05)$. None of the other biochemical variables were influenced by the number of teeth $(\mathrm{p} \geq 0.05)$. The body condition, as well as the morphometric and blood parameters, were not affected by changes in the dental timing, with the exception of serum magnesium.

Key words: buffalo, dental timing, blood biochemistry, morphometry.

\section{INTRODUCCIÓN}

En la República Argentina existe una amplia región inexplotada o explotada ineficientemente desde el punto de vista pecuario, debido a la falta de adaptación del ganado vacuno al ambiente, como son los sectores bajos e inundables que totalizan unos ocho millones de hectáreas ${ }^{14}$. Por su gran capacidad de conversión de las pasturas naturales a carne y por la perfecta coincidencia de las curvas de requerimiento con las curvas de oferta forrajera del subtrópico húmedo, la especie Bubalus bubalis representa una herramienta imbatible para la presente y futura expansión ganadera del litoral argentino.

Por su gran rusticidad ${ }^{6}$, los búfalos aprovechan mejor el forraje de baja calidad, como consecuencia de una adecuada digestión de la fibra ${ }^{29}$, aún bajo condiciones climáticas adversas, manifestando una notable resistencia a las enfermedades. La formación de los dientes se inicia entre las $6^{\circ}$ y $7^{\circ}$ semanas de desarrollo embrionario. Externamente, los dientes constan de tres partes: corona, cuello y raíz, mientras que internamente, los dientes se componen de esmalte, dentina, cemento y pulpa ${ }^{13}$.

Los principales componentes de los dientes son los cristales de hidroxiapatita (calcio), material orgánico y agua, en proporción variable de acuerdo a los componentes dentales y a las partes del diente (corona, cuello y raíz) ${ }^{15}$. Químicamente, los dientes están formados principalmente por minerales como calcio, fósforo y magnesio, entre otros, cuya movilización es regulada por factores nutricionales y endocrinos (calcitonina, parathormona, vitamina D). La formación y crecimiento de los dientes está mediada por la somatotropina y múltiples factores de crecimiento ${ }^{8}$.

La determinación de la edad en los búfalos de agua se puede establecer por los procesos de erupción, muda, desarrollo y desgaste de los dientes incisivos, lo cual es de 
vital importancia para la clasificación del ganado en sus etapas productivas y reproductivas ${ }^{16}$.

Desde su nacimiento, vacunos y bubalinos pasan por un proceso de desarrollo de sus dentaduras, presentando ciertas características que constituyen una eficiente herramienta para la estimación de la edad. Normalmente en las producciones bubalinas no se suele revisar la dentadura; esta práctica solamente se realiza cuando se requiere corroborar la edad del animal por alguna situación muy puntual.

Factores como la falta de manejo nutricional o deficiencias minerales pueden resultar en una tardía erupción dentaria e irregular desgaste ${ }^{5}$, lo que podría dificultar la correcta validación de la edad. Los valores hematológicos son una representación del estado de salud del animal y pueden ser utilizados como ayuda para evaluar el estado sanitario y nutricional del rodeo. Su importancia se centra en la comparación con valores de referencia precisos ${ }^{4}$.

El objetivo del presente trabajo fue evaluar los cambios de la arcada dentaria y número de incisivos permanentes presentes en búfalas desde los 29 hasta los 32 meses de edad, así como su correlación con la condición corporal, parámetros morfométricos y bioquímicos relacionados al metabolismo óseo, partiendo de la dentadura decidua completa.

\section{MATERIAL Y MÉTODOS}

La experiencia se llevó a cabo en un establecimiento ganadero del Departamento General Paz (Corrientes, Argentina). Se trata de una zona de relieve plano, dominada por áreas deprimidas de deficiente drenaje (bañados, esteros, cañadas). El tapiz natural predominante es de tipo pajonal con especies de mediana a baja calidad, con sobresaliente presencia de paja colorada (Andropogon lateralis) y paja amarilla (Sorghastrum agrostoides).

El clima es subtropical con una temperatura media anual $21,6^{\circ} \mathrm{C}$, una máxima de $44,9^{\circ} \mathrm{C}$ y una mínima $-1,1^{\circ} \mathrm{C}$. El régimen promedio de precipitación anual es de 1.206 $\mathrm{mm}^{1}$. Las condiciones de alimentación (a campo natural), así como la sanidad y el manejo de los animales bajo ensayo, fueron similares durante todo el ensayo.

A partir del rodeo del establecimiento, se seleccionaron treinta búfalas de raza Murrah, de 29 meses de edad, en las cuales se exploró la arcada dentaria, constatando que presentaran dentadura decidua completa, efectuándose un registro fotográfico de las mismas. Además, el día 0 se procedió a la valoración del peso vivo (PV) en báscula.

Transcurridos 3 meses (90 días), se procedió a evaluar los animales, observando la erupción de los incisivos permanentes. Además, se registró el PV, el perímetro torácico (PT, por medición con cinta métrica), la condición corporal (CC, escala 1-5, donde 1: emaciada y 5: obesa) ${ }^{28}$ y se tomaron muestras sanguíneas por venopunción yugular, para la valoración de parámetros séricos relacionados al metabolismo óseo: calcio (arsenazo III, $650 \mathrm{~nm}$ ), fósforo inorgánico (molibdato-ascorbato, $620 \mathrm{~nm}$ ), magnesio (xylidyl blue, $510 \mathrm{~nm}$ ) y fosfatasa alcalina-ALP (fenilfosfato aminoantipirina, $520 \mathrm{~nm}$ ).

Los análisis bioquímicos se llevaron a cabo con reactivos Wiener Lab, en el Laboratorio de Análisis Clínicos del Hospital Escuela de la Facultad de Ciencias Veterinarias, Universidad Nacional del Nordeste, Corrientes, Argentina.

La estadística descriptiva y el análisis de la variancia (nivel de significancia $\mathrm{p}<0,05$ ) se realizaron entre los pesos registrados a los 0 y 90 días y entre el número de incisivos presentes y las demás variables analizadas a los 3 meses de trascurrido el en- 
sayo. La diferencia entre medias se estimó por el test de Tukey. El grado de correlación entre el número de incisivos y las variables exploradas a los 90 días, se constató a través del test de Pearson. Bajo un modelo observacional descriptivo y comparativo, se examinó la cantidad de piezas dentales y su nivel de erupción.

\section{RESULTADOS Y DISCUSIÓN}

Al momento del examen inicial (día 0), todas las bubillas contaban con dentadura decidua (boca diente de leche). La bibliografía nos recuerda que tanto la especie vacuna, como la bubalina no poseen incisivos superiores. La dentición temporal o "de leche" se compone de veinte dientes, de los cuales ocho son incisivos y doce son premolares. En la dentición permanente, se suman doce molares, totalizando 32 piezas. Se considera que, para los nueve meses, el búfalo ya posee todos los dientes deciduos ${ }^{20}$.

A los 90 días, el $47 \%$ de los animales no presentó dientes incisivos permanentes erupcionados, el 37\% mostró dos dientes, mientras que el 16\% restante contó con un diente (pinza izquierda o derecha) (Figura 1). Se tuvo en cuenta el lapso de 90 días, dado que varios investigadores refieren que en la especie bubalina la erupción de los incisivos permanentes comienza en el período de los 2 a 3 años, con diferencias de meses, según los autores ${ }^{11,18,20}$.

Nuestras observaciones coinciden con otros reportes donde se comparó la erupción de los incisivos permanentes entre las especies Bos taurus, Bos indicus y Bubalus bubalis, observándose en esta última que el par de pinzas erupcionó a los 2 años y 9 meses $^{20}$.

En la especie bubalina, la dentición definitiva del primer par de incisivos surge entre 2,5 a 3 años; el segundo par, entre los 3,5 a 4 años; el tercero entre los 4 a 5 años y, finalmente, el cuarto par, aparece entre los 5 a 5,5 años ${ }^{19}$. Para otros autores, a pesar de que la formula dentaria en vacunos y bubalinos es la misma, existe una variación de la edad de aparición de los incisivos permanentes en los búfalos ${ }^{20}$.

En un estudio realizado en Colombia, se encontró que en el rango de 2 a 3 años de edad, el $60 \%$ de los animales presentaba muda de pinzas, el 10\% mudó pinzas y primeros medios, mientras que el $30 \%$ restante presentó todos los incisivos deciduos con la superficie lingual plana ${ }^{18}$. Los resultados obtenidos en dicha experiencia fueron similares a los de nuestro trabajo, mientras que difirieron con los reportados por otros investigadores que afirman que entre la edad de dos años y medio y tres años, los animales presentan las palas permanentes erupcionadas y gastadas, comenzando la erupción de los primeros medios ${ }^{11}$.

En las Figuras 2, 3 y 4 se pueden apreciar las distintas variaciones de la arcada dentaria de las bubillas, registradas en nuestra experiencia.

En los vacunos, los incisivos temporales emergen entre los dos últimos meses de gestación y las primeras semanas después del nacimiento. Sin embargo, se acepta que el número de dientes incisivos al nacer puede variar entre razas bovinas. Según este criterio, las razas vacunas pueden clasificarse como precoces (Hereford, Angus, Shorthorn), poco precoces (Holstein, Normanda) y tardías (criollos) ${ }^{3}$.

En trabajos donde se propuso determinar el número de dientes incisivos al nacer en becerros criollo limonero y las variables que lo influencian, se concluyó que dicho número parece estar más influenciado por factores no genéticos como el sexo del becerro y el número de partos de la madre, que por la precocidad racial del individuo ${ }^{27}$. 
Otros factores que pueden influir son la edad gestacional (prematuro o en término) y el peso al nacer (normal, bajo o muy bajo) ${ }^{23}$.

La única región corporal que presenta signos evidentes de paralelismo con la edad es la arcada dentaria ${ }^{17}$. Cada especie tiene una velocidad característica para su envejecimiento biológico, aunque la celeridad de la declinación funcional varía entre individuos y de órgano a órgano dentro del mismo individuo. Este hecho explica que, en los bovinos, las vacas de cría sean eliminadas del rodeo por su desgaste dental, aunque el resto del organismo pueda continuar funcionando sin inconvenientes ${ }^{9}$.

La especie bubalina tiene buenos indicadores de procreo y de ganancia de peso, en una proporción variable entre 15 y 60\% por encima a la de los vacunos. Las hembras manifiestan una destacada longevidad reproductiva pudiendo parir hasta 16 bucerros a lo largo de su vida útil, con una edad entre 18 y 22 años ${ }^{24}$.

La evolución del peso vivo de bubillas con dentadura decidua (día 0) hasta la aparición de los primeros incisivos permanentes (día 90) fueron: 422,80 \pm 40,48 kg y 430,93 \pm 44,66 kg, con una significancia de 0,462 ( $<<0,05)$.

En la Tabla 1 se consignan los resultados obtenidos para los parámetros morfométricos y bioquímicos explorados el día 90, en relación a los incisivos permanentes. Los valores de PV, PT y CC no se vieron influenciados por las modificaciones en la cantidad de piezas dentales observadas. El análisis de correlación de Pearson no arrojó valores que indicaran que estas variables estuvieran en relación a la cantidad de incisivos. Las bubillas, por lo general, alcanzan la pubertad cuando adquieren cerca del 55$60 \%$ de su peso corporal adulto (de 250 a $400 \mathrm{~kg}$ ), dependiendo éste a su vez, del genotipo animal ${ }^{22}$.

Las curvas de crecimiento de hembras bubalinas basadas en el registro del PV, revelaron que éste se estabilizó después de los 25 meses de edad. Ello reviste importancia desde el punto de vista práctico, ya que indica que, a partir de esta edad, las bubillas se pueden incorporar a la actividad reproductiva porque prácticamente ya han completado su crecimiento ${ }^{12}$.

Dentro de las variables bioquímicas relacionadas al metabolismo óseo, analizadas a los 90 días de trascurrido el ensayo, sólo los niveles séricos de magnesio manifestaron una diferencia significativa entre la cantidad de piezas dentales observadas $(p<0,05)$. La magnesemia fue mayor en los animales sin incisivos permanentes $(2,69 \pm 0,35 \mathrm{mg} / \mathrm{dl}) \mathrm{y}$ de un diente $(2,66 \pm 0,50 \mathrm{mg} / \mathrm{dl})$ y menor en los de dos dientes $(2,27 \pm 0,38 \mathrm{mg} / \mathrm{dl})$, revelando una correlación negativa $(-0,45 ; \mathrm{p}<0,05)$ entre este parámetro y el número de incisivos. Los valores hallados se encontraron dentro de los citados en la bibliografía para vacunos $(2,4 \text { a } 3,3 \mathrm{mg} / \mathrm{dl})^{8}$.

Los niveles séricos de calcio registrados en nuestro trabajo, se encontrarían dentro de los rangos reportados para vacas $(9,4 \text { a } 10,5 \mathrm{mg} / \mathrm{dl})^{8}$. La fosfatemia revelada por las búfalas en nuestra experiencia, se mostró más elevada que las citados en la bibliografía para vacas, 4 a $5 \mathrm{mg} / \mathrm{dl}^{8}$. En bovinos jóvenes, este parámetro puede presentarse más elevado que en los adultos ${ }^{7}$.

Los cambios en la composición mineral del tejido dental, pueden estar asociados con trastornos metabólicos que modifiquen el peso corporal de los individuos ${ }^{16}$. Un estudio reveló que la ultraestructura del esmalte presenta una similitud significativa entre las especies humana, vacuna y bubalina. En cuanto a la microdureza no hubo diferencias estadísticamente significativas entre las tres especies estudiadas. Sin embargo, la rugosidad de la superficie del esmalte de los búfalos $(2,16 \pm 0,23 \mu \mathrm{m})$ fue significativa- 
mente mayor, en comparación con los dientes humanos $(0,36 \pm 0,05 \mu \mathrm{m})$ y vacunos $(0,41 \pm 0,07 \mu \mathrm{m})^{21}$.

En animales, factores como la falta de aporte nutricional y la deficiencia mineral, pueden resultar en una erupción tardía y en el desgaste irregular de la arcada dentaria ${ }^{5}$ lo que dificultaría la evaluación correcta de la edad, sobre todo en épocas de muda y desgaste dental ${ }^{25}$.

En un trabajo cuyo objetivo fue evaluar los parámetros sanguíneos de búfalas de Rumania, se obtuvieron valores promedios de 92-171 U/l de ALP; 5,0-9,3 mg/dl de calcio total; 1,3-3,9 mg/dl de fósforo inorgánico y 1,27-3,17 mg/dl de magnesio. Al considerar estas variables de acuerdo al estado fisiológico de los animales (hembras preñadas y hembras en lactación), los niveles fueron ALP: 147,0 \pm 24,71 y 103,50 \pm 11,36 UI/l, calcio: 8,48 \pm 0,43 y 6,48 \pm 1,92 mg/dl, fósforo inorgánico: 2,68 $\pm 1,26$ y 2,28 $\pm 0,92$ $\mathrm{mg} / \mathrm{dl}$ y magnesio: $2,92 \pm 0,20$ y $2,03 \pm 0,66 \mathrm{mg} / \mathrm{dl}$, respectivamente. Los autores, hallaron que ALP fue significativamente más baja en el grupo de búfalas lactando, lo cual también fue observado en los electrolitos evaluados ${ }^{26}$. En relación a nuestra experiencia, los valores citados fueron menores, a excepción del magnesio, que guardó cierta similitud.

En la evaluación sanguínea de búfalos de raza Murrah de Brasil, separados en tres grupos según su edad: de 2 a 8 meses, de 9 meses a 2 años y animales mayores a 2 años, se reportaron valores de ALP de 307,43 \pm 124,22; 356,0 \pm 153,42; y 179,7 \pm 62,32 UI/l, respectivamente ${ }^{10}$. Las mayores concentraciones séricas de esta enzima, se registrarían en animales jóvenes y se deberían a la isoenzima ósea, reveladora de una elevada actividad osteoblástica y crecimiento óseo ${ }^{7}$.

Un estudio relacionó el perfil mineral sérico de búfalas que padecían hemoglobinuria post-parto, con el perfil mineral de suelos y forrajes de las áreas propensas a la enfermedad. Tal enfermedad es aguda, se presenta tanto en búfalas como en vacas lecheras y se caracteriza por hipofosfatemia, hemólisis intravascular, hemoglobinuria y anemia. Estos autores, citan valores de calcemia de 9,83 mg/dl para los animales sanos y de 9,86 mg/dl para aquéllos con síntomas de anemia. Una diferencia manifiesta se halló en los niveles de fósforo, que fueron significativamente bajos en las hembras anémicas $(1,88 \mathrm{mg} / \mathrm{dl})$ con relación a las sanas $(5,41 \mathrm{mg} / \mathrm{dl})$. Además, se halló una marcada relación entre hipofosfatemia sérica con el escaso fosfato detectado en los suelos (provincia de Punjab, Pakistán) y en los alimentos que formaban parte de la ración de los animales ${ }^{2}$. Cabe señalar que los valores de fósforo citados para las hembras sanas, tienen similitud con los resultados obtenidos en nuestra experiencia.

Otra enfermedad que padecen las búfalas lecheras de alta producción es la cetosis. Clínicamente los cuadros agudos se caracterizan por anorexia, constipación, disminución de la producción láctea, distensión ruminal y pérdida marcada de la condición corporal, con hipofosfatemia, hipomagnesemia e hipercalcemia. En una experiencia, se trabajó con tres grupos de animales: búfalas clínicamente sanas, animales que padecían cetosis subclínica y un tercer grupo de hembras con sintomatología de cetosis aguda. Los valores de calcio fueron de 8,9 \pm 0,9; 10,3 $\pm 3,0$ y 10,1 $\pm 2,6 \mathrm{mg} / \mathrm{dl}$; los de fósforo fueron de $5,8 \pm 1,15 ; 4,1 \pm 2,69$ y 3,6 $\pm 1,5 \mathrm{mg} / \mathrm{dl}$ y los de magnesio fueron de $1,1 \pm$ 0,$17 ; 0,9 \pm 0,58$ y $1,0 \pm 0,1 \mathrm{mg} / \mathrm{dl}$ respectivamente ${ }^{30}$.

En conclusión, las variables morfométricas y bioquímicas exploradas no se vieron afectadas por las modificaciones de la cronometría dentaria, registrada a los 90 días de transcurrido el ensayo, a excepción del magnesio sérico. 
Agradecimientos. A Nilda Lotero y Horacio Maier, por permitir el uso de su establecimiento y participar activamente en el desarrollo del ensayo. A Wiener Lab (Rosario, Argentina), por la provisión de los reactivos bioquímicos utilizados.

\section{REFERENCIAS}

1. Acosta F, Gimenez L, Richieri C, Calvi M. 2009. Zonas agroeconómicas homogéneas Corrientes: descripción ambiental, socioeconómica y productiva. Publ. Centro Regional INTA Corrientes, Argentina. ISSN 1851-6955.

2. Akhtar MZ, Khan A, Sarwar M, Javaid A. 2007. Influence of soil and forage minerals on buffalo (Bubalus bubalis) parturient haemoglobinuria. Asian-Aust J Anim Sci 20: 393-398.

3. Arzalluz A. 1992. Determinación del número de incisivos en bovinos mestizos al nacimiento en la región zuliana. Trabajo de ascenso, Facultad de Ciencias Veterinarias, Universidad del Zulia, Venezuela, $42 \mathrm{p}$.

4. Beechler BR, Jolles AE, Ezenwa O. 2009. Evaluation of hematologic values in freeranging african buffalo. $J$ Wild Dis 45: 57-66.

5. Blood DC, Henderson JA, Radostits OM. 1983. Clínica veterinária, $5^{\circ}$ ed., Ed. Guanabara-Koogan, Rio de Janeiro, p.15.

6. Cockrill WR. 1980. The ascendant water buffalo-key domestic animal. World Anim Rev 33: 2-13.

7. Coles EH. 1989. Veterinary clinical pathology, Ed. Saunders, Philadelphia, USA, $496 \mathrm{p}$.

8. Coppo JA. 2008. Fisiología comparada del medio interno, Ed. Eucasa, Salta, Argentina, $310 \mathrm{p}$.

9 Facio F. 1982. Prótesis dental en bovinos. Rev Anales Soc Rural Arg, Buenos Aires, 82: $18-22$.

10. Fontes D et al. 2014. Perfil hematológico e bioquímico de búfalos (Bubalus bubalis) na Amazônia Oriental. Pesq Vet Bras 34: 57-63.

11. Frison G, Reher C. 1980. Age determination of buffalo by tooth eruption and wear. Plains Anthropol 7: 46-50.

12. Fundadora O. 2015. Comportamiento de búfalos de agua (Bubalus bubalis) de la raza Buffalypso. Rev Cubana Cienc Agríc 49: 161-171.

13. Gartner LD, Hiatt LJ. 1997. Sistema digestivo I: cavidad oral. En: Histología, Capítulo 16, Ed. McGraw-Hill, 2da ed., México, p. 320-330.

14. Gerometta J. 2015. La cría de búfalos se consolida en la región con ventajas competitivas. www.diarionorte.com/article/125668/.

15. Jain MK, Sing NP, Dave BK. 2001. Age determination in crossbred calves using different techniques. Indian J Anim Sci 71: 1058-1061.

16. Luengo LJ, Aros IC, Gómez RL. 1990. Determinación de la edad del bovino según las características morfológicas de los dientes incisivos. Avan Cien Vet (Chile), 5: 1. doi: 10.5354/0719-5273.1990.10398.

17. Martínez RD, Fernández EN, Abbiati NN. 2011. Desgaste dental de los bovinos. Rev Vet Arg 28: 277. Recuperado de www.produccion-animal.com.ar

18. Martínez VE, Fernández CN. 2012. Determinación de edad mediante cambios en piezas dentales en búfalos de agua (Bubalus bubalis). Trabajo de grado, Universidad de La Salle, Fac. Cs. Agrop., Bogotá, Colombia. 
19. Miranda WC. 1986. Criação de búfalo no Brasil, Edit. Dos Criadores, São Paulo, Brasil, 173 p.

20. Moran JB. 1992. Growth and development of buffaloes. In: Buffalo production (Tulloch D and Holmes CW Ed.), Elsevier (Sidney, Australia), p. 199-221.

21. Nogueira BC et al. 2014. Avaliação comparativa da ultraestrutura e propiedades físicas do esmalte bovino, bubalino e humano. Pesq Vet Bras 34: 485-490.

22. Perera BM. 2010. Reproductive cycles of buffalo. Anim Reprod Sci. doi:10.1016/ j.anireprosci.2010.08.022.

23. Ramos SR, Gugisch RC, Fraiz FC. 2006. The influence of gestation alage and birth weight of the newborn on tooth eruption. J Appl Oral Sci 14: 228-232

24. Sabatini DJ. 2014. Producción animal no tradicional. Public. Ministerio de Ciencia, Tecnología e Innovación Productiva, Buenos Aires, Argentina.

25. Santos FC et al. 2008. Análise morfológica dos dentes incisivos de búfalos e sua relação com a idade de abate. Ciên Anim Bras 9: 506-511.

26. Serdaru1 M et al. 2011. Seasonal variations of some hematological and biochemical parameters of the carpathian romanian buffaloes. Anim Sci \& Biotechnol 44: 9498

27. Urdaneta $\mathbf{M}$ et al. 2008. Factores que afectan el número de dientes incisivos al nacer en becerros criollo limonero. Desgaste dentario hasta los 12 meses de edad. Rev Científ (FCV-LUZ), 18: 542-548.

28. Van Niekerk A, Louw BP. 1980. Condition scoring of beef cattle. Bulletin N 2/80. Department of Agriculture and Fisheries, Natal Region, South Africa.

29. Wanapat M, Chanthakhoun V. 2009. Recent advances in rumen ecology, digestion and feeding strategies of swamp buffaloes. America's Buffalo Symposium, Proceeding 4: 27.

30. Youssef MA, Khodery SA, Deeb WM, Abou WE. 2010. Ketosis in buffalo (Bubalus bubalis): clinical findings and the associated oxidative stress level. Trop Anim Health Prod 42: 1771-1777.

Tabla 1. Variaciones de los parámetros relacionados al metabolismo óseo en bubillas con relación al número de incisivos permanentes.

\begin{tabular}{lccccc}
\hline \multicolumn{1}{c}{ variables } & incisivos (D) & incisivo (uno) & incisivos (par) & $\overline{\mathrm{x}} \pm \mathrm{DE}$ & $\mathrm{p}$ \\
\hline PV $(\mathrm{kg})$ & $425,36 \pm 16,13$ & $456 \pm 17,67$ & $426,64 \pm 12,91$ & $430,9 \pm 44,6$ & 0,401 \\
PT $(\mathrm{cm})$ & $190 \pm 7,66$ & $197,20 \pm 4,76$ & $189,91 \pm 8,94$ & $191,1 \pm 8,03$ & 0,187 \\
CC $($ score) & $3,32 \pm 0,25$ & $3,60 \pm 0,55$ & $3,32 \pm 0,40$ & $3,37 \pm 0,37$ & 0,313 \\
Ca (mg/dl) & $10,85 \pm 0,76$ & $10,20 \pm 1,03$ & $10,37 \pm 0,74$ & $10,57 \pm 0,82$ & 0,198
\end{tabular}




\begin{tabular}{lccccc}
$\mathrm{P}(\mathrm{mg} / \mathrm{dl})$ & $5,94 \pm 1,02$ & $5,78 \pm 0,78$ & $5,43 \pm 0,83$ & $5,73 \pm 0,92$ & 0,385 \\
$\mathrm{Mg}(\mathrm{mg} / \mathrm{dl})$ & $2,69 \pm 0,35$ & $2,66 \pm 0,50$ & $2,27 \pm 0,38$ & $2,53 \pm 0,42$ & $0,32 *$ \\
$\mathrm{ALP}(\mathrm{UI} / \mathrm{l})$ & $197 \pm 41$ & $223 \pm 39$ & $214 \pm 31$ & $207 \pm 58$ & 0,632 \\
\hline
\end{tabular}

D: deciduo, $\overline{\mathrm{X}}$ : media aritmética, DE: desvío estándar, PV: peso vivo, PT: perímetro torácico, CC: condición corporal, Ca: calcio, P: fósforo, Mg: magnesio, ALP: fosfatasa alcalina, * significancia: p<0,05.

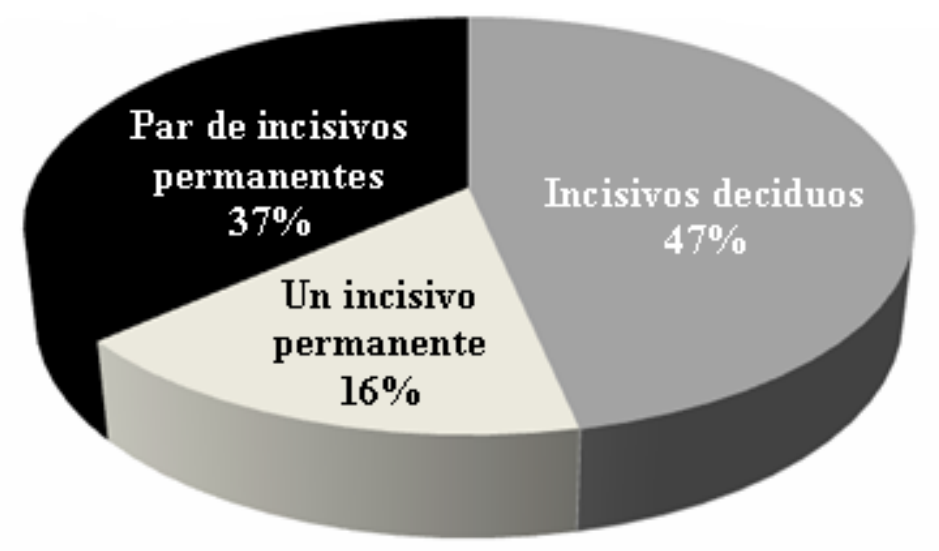

Figura 1. Distribución de los porcentajes de incisivos permanentes en las bubillas.

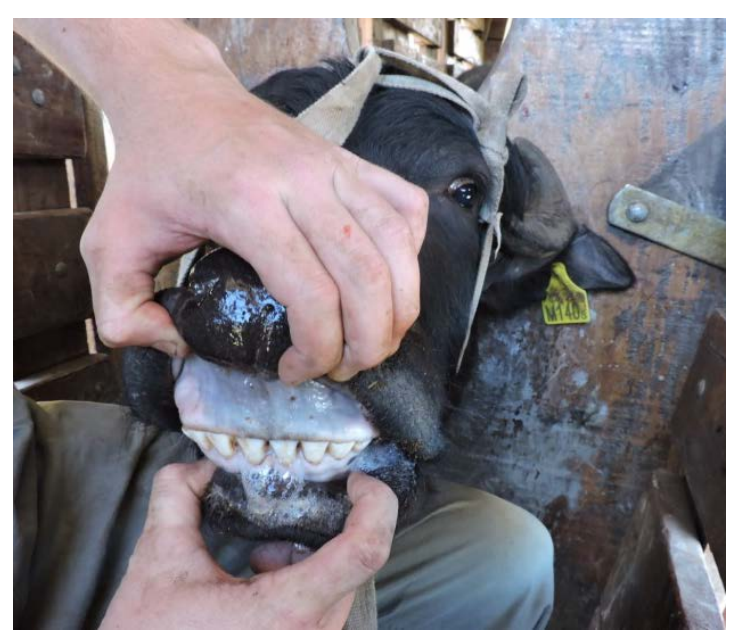

Figura 2. Ausencia de dientes permanentes. 


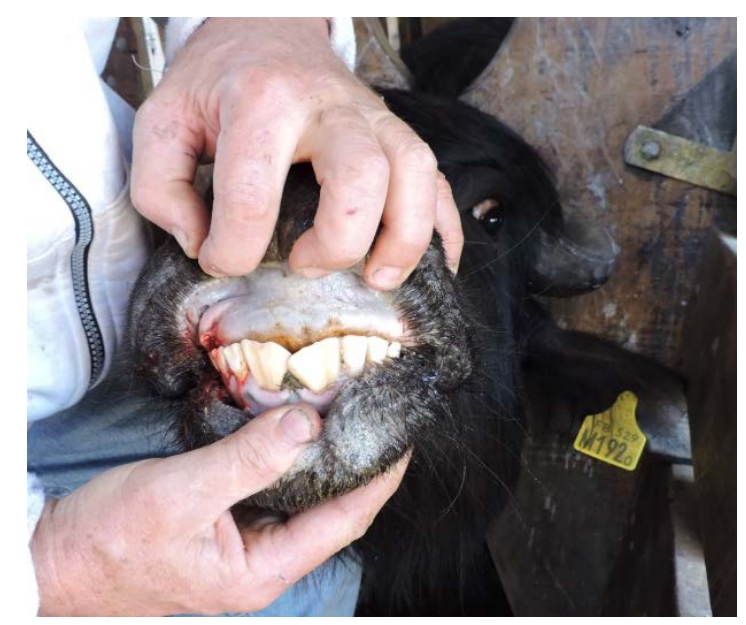

Figura 3. Presencia de ambas pinzas permanentes.

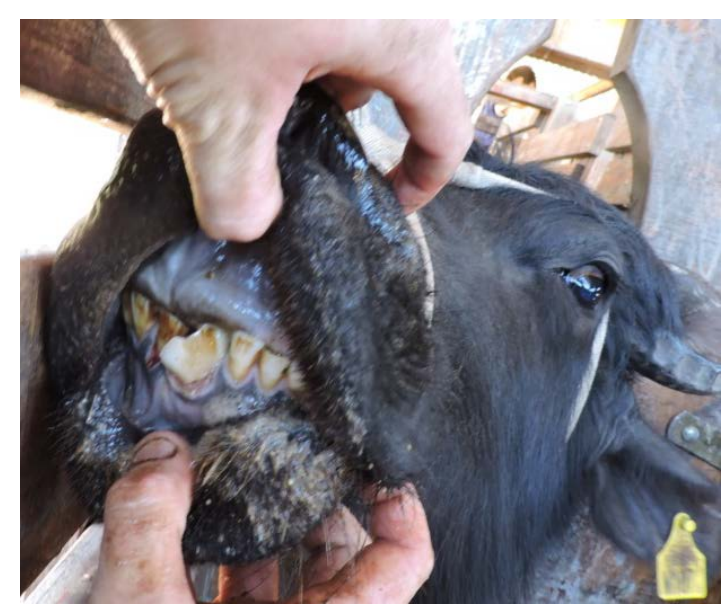

Figura 4. Erupción de la pinza izquierda. 